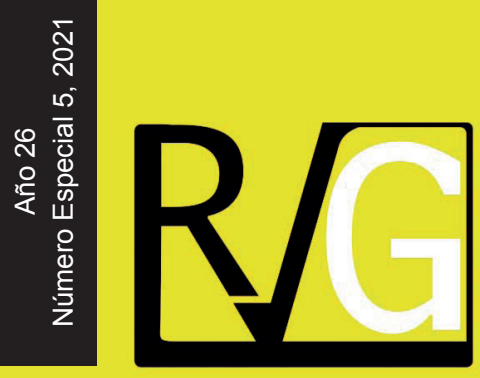

\title{
Año 26
}

\section{Número Especial 5, 2021}

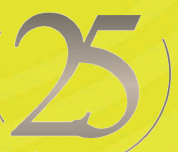

ANMERSARO
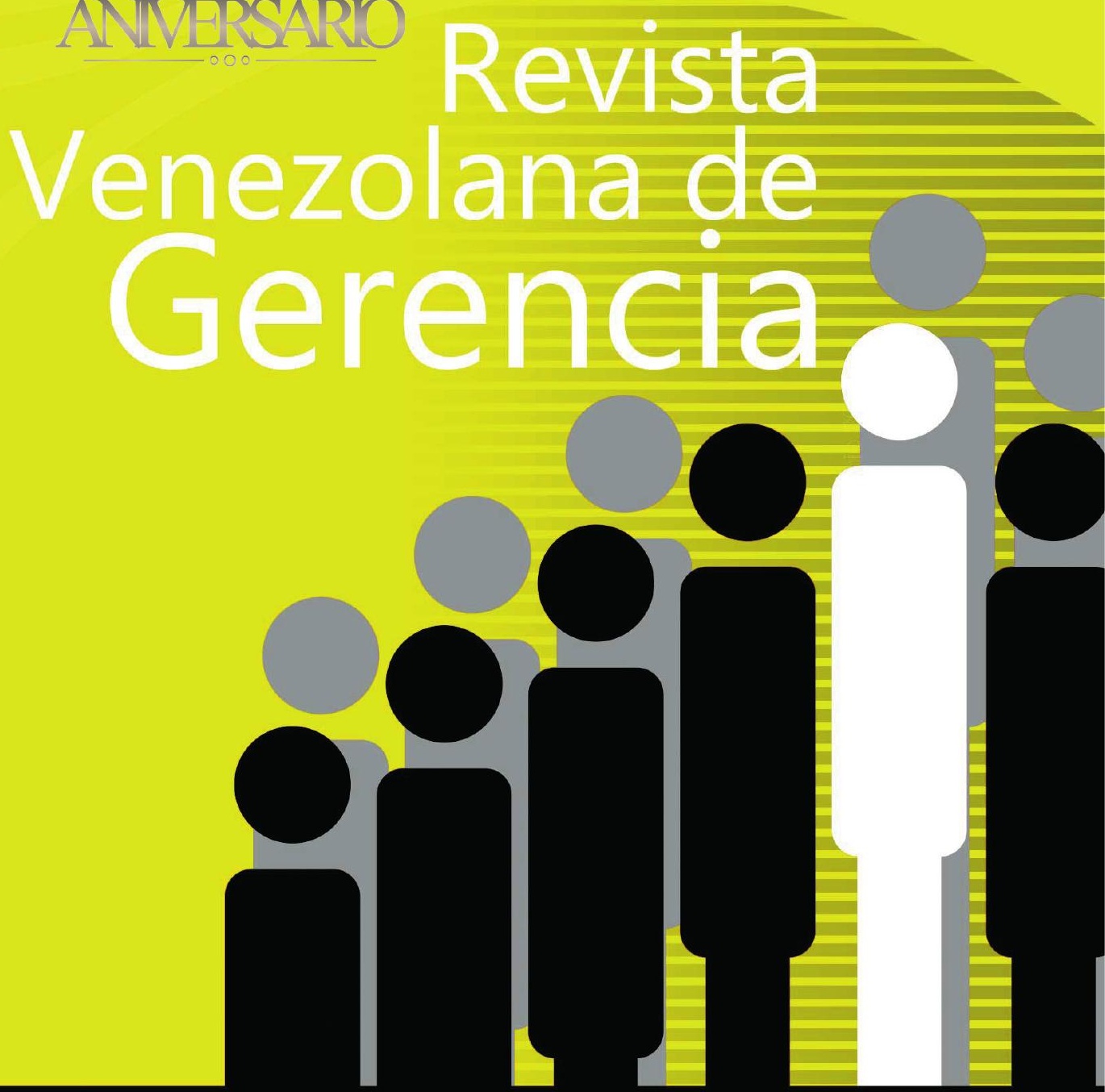

UNIVERSIDAD DEL ZULIA (LUZ)

Facultad de Ciencias Económicas y Sociales

ISSN 1315-9984

Centro de Estudios de la Empresa

Esta obra está bajo una licencia de Creative Commons 


\title{
Calidad de servicio en tiempos de Covid-19 en estudiantes universitarios
}

\author{
Neyra-Huamani, Lidia* \\ Flores-Morales, Jorge Alberto** \\ Pacheco-Pomarino, Mariluz***
}

\section{Resumen}

La enfermedad del coronavirus COVID-19 ha cambiado la forma de educación de presencial a remoto con el propósito de reducir el contagio en los estudiantes de todos los niveles educativos. El objetivo principal del presente estudio fue determinar el nivel de percepción de calidad de servicio en tiempos de pandemia en estudiantes de una universidad nacional de Lima. La muestra fue intencional conformada por 142 estudiantes de educación. El diseño es no experimental descriptiva, de enfoque cuantitativo y tipo de investigación Básica. El instrumento empleado fue una adaptación del cuestionario SERVQUAL sobre calidad de servicios y los datos recolectados fueron analizados mediante el programa estadístico SPSS. Los resultados del estudio evidencian un nivel alto de percepción con un $66.9 \%$ sobre los servicios que presta la universidad a los estudiantes y la rapidez como ésta se adecuo a una educación remota marcada por el coivd-19. Se concluye que es importante medir siempre la calidad de servicio educativo y más una en estos tiempos de pandemia, para poder hacer las modificaciones debidas o a tiempo y mantener el buen ambiente de la comunidad universitaria expresada en la virtualidad.

Palabras clave: calidad de servicio; educación virtual; estudiantes; pandemia; universidad.

\section{Recibido: 15.01 .21}

Aceptado: 20.05 .21

* Doctora y Magister en Ciencias de la Educación, Universidad Nacional Enrique Guzmán y Valle. Licenciada en Educación, Universidad Marcelino Champagnat. Docente de la Universidad Tecnológica del Perú. Investigadora RENACYT N P0024288. Perú. E-mail: C18727@utp.edu.pe ORCID: https://orcid.org/00000001-6261-2190

** Doctor en Psicología y Educación por la Universidad César Vallejo. Magister en Educación. Licenciado en Psicología por la Universidad Federico Villarreal. Docente de la Universidad Femenina del Sagrado Corazón UNIFÉ, Perú. E-mail: jorgefloresm@unife.pe. ORCID: https://orcid.org/0000-0002-3678-5511

*** Magister en Docencia Universitaria, Universidad Nacional Enrique Guzmán y Valle. Licenciada en Educación artística, Universidad Mayor de San Marcos. Docente de la Universidad Nacional Enrique Guzmán y Valle.

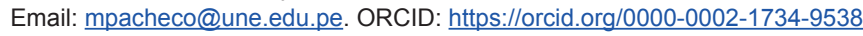




\title{
Quality of service in students of a national university in Lima, 2020
}

\begin{abstract}
The coronavirus disease COVID-19 has changed the form of education from face-toface to remote in order to reduce the contagion in students of all educational levels. The main objective of this study was to determine the level of perception of service quality in times of pandemic in students of a national university in Lima. The sample was intentional made up of 142 education students. The design is non-experimental, descriptive, with a quantitative approach and a Basic type of research. The instrument used was an adaptation of the SERVQUAL questionnaire on quality of services and the data collected were analyzed using the SPSS statistical program. The results of the study show a high level of perception with $66.9 \%$ of the services provided by the university to students and how quickly it was adapted to a remote education marked by the COIVD-19. It is concluded that it is important to always measure the quality of educational service and more one in these times of pandemic, to be able to make the modifications due or on time and maintain the good atmosphere of the university community expressed in virtuality.
\end{abstract}

Key words: Quality of service; virtual education; students; pandemic; university.

\section{Introducción}

La pandemia provocada por el covid-19 no solo afectó en el área de la salud sino también a la economía, los planes de gobierno, el deporte, el turismo, etc. El sistema educativo también fue afectado y tuvo que tomar las medidas adecuadas para no desatender la formación educativa de niños y jóvenes cambiando de modalidad de enseñanza. Por esta razón, la recomendación de las autoridades sanitarias obligó al sector educativo a buscar una alternativa al método presencial (Dwidienawati, et al, 2020). Asimismo, la pandemia del COVID-19 ha transformado los procesos de formación y la transición de la presencialidad a la virtualidad ha afectado a todo el proceso educativo. Ello, ha favorecido uno de los procesos de innovación: la capacidad de gestionar el flujo de conocimiento en toda las áreas (Tejedor, et al, 2021).

En la esfera de la educación, esta emergencia ha dado lugar al cierre masivo de las actividades presenciales de instituciones educativas en más de 190 países con el fin de evitar la propagación del virus y mitigar su impacto (CEPAL-UNESCO, 2020). Obviamente, para mitigar la transmisión del COVID-19, los cambios rápidos en la impartición de la enseñanza se trasladaron de la instrucción presencial a la remota (Moser, Wei, \& Brenner, 2021).

En este contexto de cambios por la pandemia del COVID-19, el sistema de enseñanza superior tuvo que adaptarse rápidamente para seguir ofreciendo 
Neyra-Huamani, Lidia; Flores-Morales, Jorge Alberto; Pacheco-Pomarino, Mariluz Calidad de servicio en tiempos de Covid-19 en estudiantes universitarios

con calidad los servicios educativos y los estudiantes no se atrasen en sus estudios manteniendo sus aspiraciones profesionales. Ello no ha sido fácil ya que se tuvo que enfrentar en un primer momento la "falta de recursos necesarios -instalaciones de aprendizaje electrónico, aulas, centros informáticos, laboratorios, etc.-", "falta de planificación estratégica" y "falta de recursos financieros" (Kinker, Swarnakar, Singh, \& Jain, 2020).

En este proceso de adecuación muchas universidades tuvieron que invertir en sus instalaciones para mantener la calidad de servicio y atender a los estudiantes de manera virtual no solamente a través del proceso enseñanza-aprendizaje que es una de las principales actividades del sistema universitario, sino también en la atención de otros servicios de igual de importantes como son la atención administrativa a docentes y alumnos.

Así que, es necesario conocer que tanto perciben los estudiantes sobre la calidad de servicio de la universidad en estos tiempos de pandemia explorando las dimensiones de fiabilidad, capacidad de respuesta tangibilidad, seguridad y empatía que estas puedan brindar a sus estudiantes.

\section{Calidad de servicio: reflexiones teóricas}

La calidad del servicio es vista como una función de la diferencia entre las expectativas del cliente y las percepciones del rendimiento del servicio (Parasuraman, Zeithaml, \& Berry, 1985). Asimismo, la calidad percibida de un servicio como una función de la brecha entre las expectativas de los consumidores sobre el servicio y sus percepciones del servicio real prestado por la organización, y sugirieron que esta brecha está influida por varias otras discrepancias o brechas que pueden darse dentro de la organización (Lewis \& Mitchell, 1990)

El modelo SERVQUAL es un método de investigación que goza de reconocimiento $y$ por ende es ampliamente utilizado para evaluar la calidad de los servicios tanto en el campo empresarial como en el académico (Parasuraman, Zeithaml, \& Berry, 1988). En función a este modelo los autores consideraron las cinco dimensiones de la calidad del servicio (Parasuraman et al,1985), (cuadro 1).

\section{Cuadro 1}

\section{Dimensiones de calidad de servicio SERVQUAL}

\begin{tabular}{ll}
\hline Dimensiones SERVQUA & \multicolumn{1}{c}{ Conceptualización } \\
\hline Elementos tangibles & $\begin{array}{l}\text { Apariencia de las instalaciones físicas, equipos, empleados y materiales de co- } \\
\text { municación. }\end{array}$ \\
\hline Fiabilidad & Capacidad para ejecutar el servicio prometido de forma segura y precisa \\
\hline Capacidad de respuesta & $\begin{array}{l}\text { Rapidez de la atención al cliente y } \\
\text { predisposición de ayudar. }\end{array}$ \\
\hline Seguridad & $\begin{array}{l}\text { Capacidad de la empresa y sus empleados para inspirar al cliente credibilidad, } \\
\text { mediante el conocimiento y cortesía }\end{array}$ \\
\hline Empatía & $\begin{array}{l}\text { Atención personalizada gracias al entendimiento de las necesidades y deseos } \\
\text { del cliente. }\end{array}$
\end{tabular}

Fuente: Parasuraman, Zeithaml \& Berry (1985) 
Además SERVQUAL es el modelo más utilizado a la hora de medir la calidad del servicio (Yarimoglu, 2014). Pues bien, la calidad de servicio es considerada como la medida en que un servicio satisface las necesidades o expectativas de los clientes (Lewis \& Mitchell, 1990). En este sentido, la calidad de servicio en las organizaciones radica en orientar a los clientes en lograr cumplir con una necesidad, y además facilitarle el cumplimiento de sus expectativas individuales (RojasMartínez, et al, 2020).

La calidad del servicio se define como la diferencia entre las expectativas de los clientes respecto del servicio y el servicio percibido. Si las expectativas son mayores que el rendimiento, entonces la calidad percibida es menos que satisfactoria y, por lo tanto, se produce insatisfacción del cliente (Parasuraman et al,1985)

Asimismo, desde la perspectiva del "mejor valor", la medición de la calidad del servicio en el sector público debe tener en cuenta las expectativas de los clientes en cuanto al servicio, así como las percepciones del mismo y el (Wisniewski, 2001). En este sentido, la calidad de servicio es un indicador clave para la gestión de las instituciones de educación superior (Mejías, Valle, \& Vega, 2016). Naturalmente, se cree que los servicios de calidad apoyan el éxito de las actividades de aprendizaje y mejoran la satisfacción de los usuarios (Napitupulu et al, 2018)

Una de las evidencias de la calidad de servicio es la competitividad de las instituciones de enseñanza. El cual, está determinada por su capacidad y prontitud para adaptarse a las necesidades cambiantes del entorno socioeconómico, y esto último sólo puede lograrse impartiendo una enseñanza de calidad y mejorándola continuamente (Datta \& Vardhan, 2017).

La calidad de los servicios tiene un impacto significativo en el rendimiento de las empresas. A través de este resultado, la empresa debe sostener la innovación, la operación de orientación al cliente. Estas actividades tienen un impacto significativo en la calidad del servicio. La innovación de la orientación al cliente no es un problema de opción sino de supervivencia (Tan \& Goh, 2017).

\section{Aspectos Metodológicos de la investigación}

El presente estudio es cuantitativo que se circunscribe dentro del paradigma positivista. El tipo de investigación es básica y de diseño descriptivo. Este tipo de estudios descriptivos tienen como finalidad especificar propiedades y características de conceptos, fenómenos, variables 0 hechos en un contexto determinado (Hernández \& Mendoza, 2018). En este caso del estudio se pone a consideración la teoría de la calidad de servicio en el entorno académico con los acontecimientos actuales producto del covid-19. En el estudio se describe la percepción de los estudiantes sobre calidad de servicio.

Se empleó el Cuestionario SERVQUAL adaptado para recoger los datos sobre calidad de servicio. Este instrumento, consta de 22 ítems. Cada ítem, está valorada a través de 
Neyra-Huamani, Lidia; Flores-Morales, Jorge Alberto; Pacheco-Pomarino, Mariluz Calidad de servicio en tiempos de Covid-19 en estudiantes universitarios

una escala de Likert $1-5$. El puntaje es de 1 a 5 puntos. El puntaje global mínimo es de 22 puntos y el puntaje global máximo es de 110 puntos. El cuestionario mide las siguientes dimensiones: Fiabilidad, Seguridad, Elementos tangibles, Capacidad de respuesta y Empatía (Parasuraman et al,1985). Este instrumento adaptado fue validado por expertos. Además, cuenta con la confiabilidad de 0,78 . Para ello se empleó Alpha de Cronbach.

La técnica de recogida de datos fue el cuestionario online a través del Google form. Con este cuestionario se recogieron los datos de octubre a diciembre de 2020. Este cuestionario respondió 142 estudiantes de la universidad nacional de Lima, 2020. 41 alumnas que representa el $29 \%$ mujeres y 101 alumnos que representa $71 \%$. Los 142 estudiantes fueron seleccionados de acuerdo al muestro aleatorio simple (Hernández \& Mendoza, 2018). cual, corresponde a los estudiantes matriculados en pregrado en el periodo lectivo 2020-II.

\section{Dimensiones consideradas de la calidad de servicio en estudiantes universitarios: contexto pandemia}

En la tabla 1 la variable Calidad de servicio, de 142 estudiantes que representan el $100 \%$, se evidencia que 95 estudiantes alcanzaron el nivel alto en cuanto a la satisfacción de la calidad de servicio representado un 66,90 \% de la población total. Así mismo, 47 estudiantes que representan un 33,1. \% perciben que la calidad de servicio que presta la universidad se encuentra en un nivel promedio. En esta variable ninguno de los estudiantes se encuentra en el nivel bajo concentrándose la mayoría en el nivel alto en la percepción de la calidad de servicio.

\section{Tabla 1}

Variable: Calidad de Servicio

\begin{tabular}{|c|c|c|c|c|c|}
\hline & & Frecuencia & Porcentaje & Porcentaje válido & Porcentaje acumulado \\
\hline \multirow{3}{*}{ Válido } & Medio & 47 & 33,1 & 33,1 & 33,1 \\
\hline & Alto & 95 & 66,9 & 66,9 & 100,0 \\
\hline & Total & 142 & 100,0 & 100,0 & \\
\hline
\end{tabular}

Fuente: Elaboración propia (2021)

En este sentido el gráfico 1 representa los datos sobre la Calidad de servicio percibida por los estudiantes universitarios, donde el mayor porcentaje se encuentra en el nivel alto con un $66,90 \%$ 


\section{Gráfico 1 \\ Histograma de la variable Calidad de servicio}

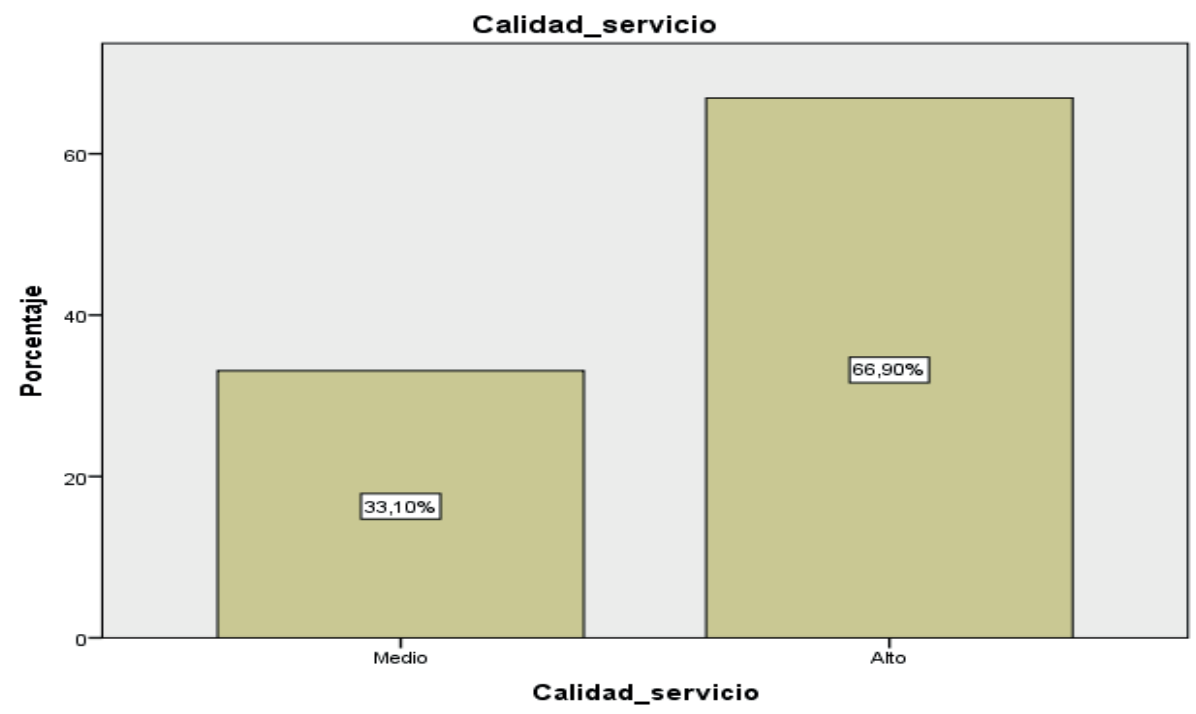

Fuente: Elaboración propia (2021)

En la tabla 2 la dimensión Tangibilidad, de los 142 estudiantes que representan el $100 \%$, se evidencia 74 estudiantes consideran que el aspecto Tangible de la Calidad de servicio en la universidad se encuentra en un nivel promedio representado un $52,1 \%$. Asimismo, 64 estudiantes consideran que se encuentran en un nivel alto con un $45,1 . \%$ y 4 estudiantes lo perciben en un nivel bajo con un $2,8 \%$ en la dimensión tangibilidad.

\section{Tabla 2}

\section{Dimensión 1: Tangibilidad}

\begin{tabular}{|c|c|c|c|c|c|}
\hline & & Frecuencia & Porcentaje & Porcentaje válido & $\begin{array}{l}\text { Porcentaje } \\
\text { acumulado }\end{array}$ \\
\hline \multirow[t]{4}{*}{ Válido } & Bajo & 4 & 2,8 & 2,8 & 2,8 \\
\hline & Medio & 74 & 52,1 & 52,1 & 54,9 \\
\hline & Alto & 64 & 45,1 & 45,1 & 100,0 \\
\hline & Total & 142 & 100,0 & 100,0 & \\
\hline
\end{tabular}

Fuente: Elaboración propia (2021) 
Neyra-Huamani, Lidia; Flores-Morales, Jorge Alberto; Pacheco-Pomarino, Mariluz Calidad de servicio en tiempos de Covid-19 en estudiantes universitarios

El gráfico 2 representa los datos de la dimensión Tangibilidad en la Calidad de servicio percibida por los estudiantes universitarios, donde el mayor porcentaje se encuentra en el nivel promedio con un $52,11 \%$.

\section{Gráfico 2}

\section{Histograma de la dimensión Tangibilidad}

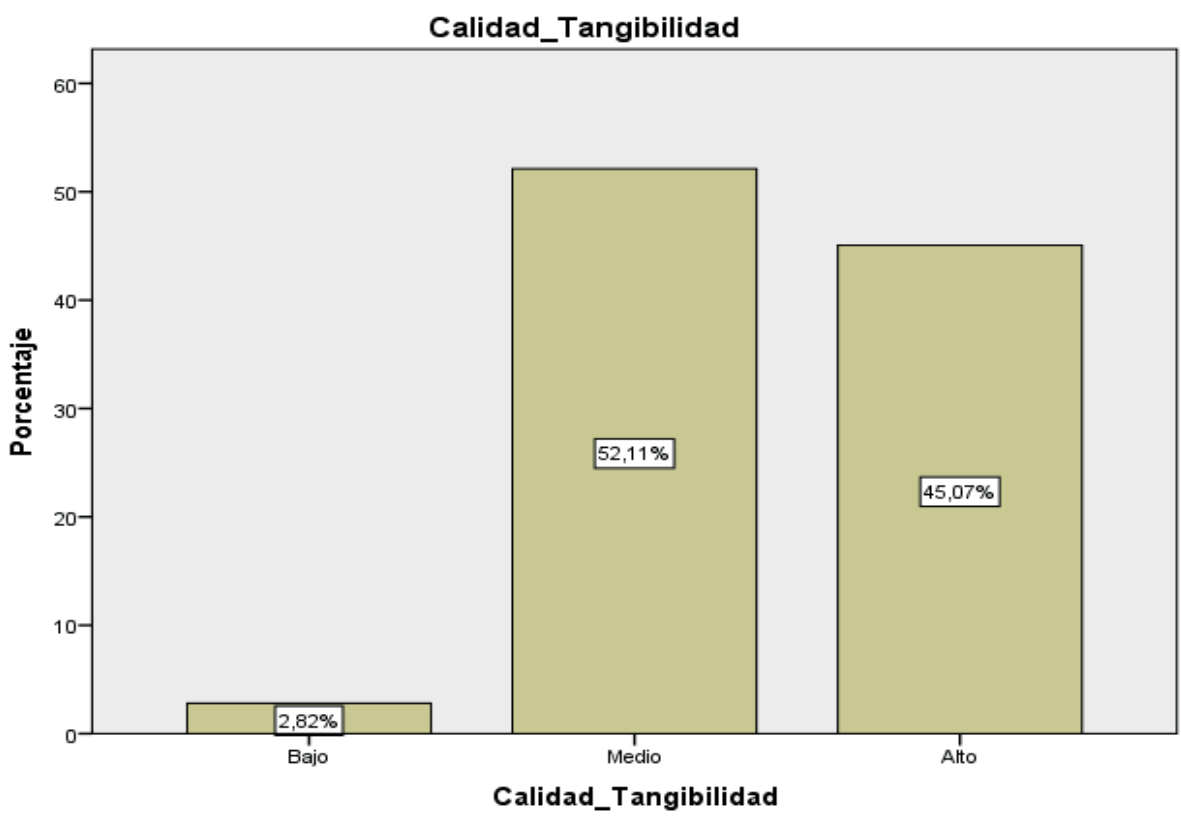

Fuente: Elaboración propia (2021)

En la tabla 3 la dimensión Fiabilidad de 142 estudiantes que representan el $100 \%$ se evidencia 101 estudiantes consideran que es Fiable o de confianza la Calidad de servicio que presta la universidad obteniendo un porcentaje de $71,1 \%$ en el nivel alto.
Además, 40 estudiantes lo consideran que se encuentra en un nivel promedio con un $28.2 \%$ se encuentran en el nivel medio con un $28,2 \%$ y un estudiante lo percibe en un nivel bajo con un $0,7 \%$ en la dimensión fiabilidad. 
Tabla 3

Dimensión 2: Fiabilidad

\begin{tabular}{llrrrrr}
\hline & & & & & \multicolumn{2}{c}{$\begin{array}{c}\text { Porcentaje acu- } \\
\text { mulado }\end{array}$} \\
\hline Válido & \multicolumn{1}{c}{ Bajo } & 1 & \multicolumn{1}{c}{ Porcentaje } & Porcentaje válido & 7 \\
\cline { 2 - 7 } & Medio & 40 & 28,2 & 7 & 28,9 \\
\cline { 2 - 7 } & Alto & 101 & 71,1 & 71,1 & 100,0 \\
\cline { 2 - 7 } & Total & 142 & 100,0 & 100,0 & \\
\hline
\end{tabular}

Fuente: Elaboración propia (2021)

El gráfico 3 representa los datos de la dimensión Fiabilidad en la Calidad de servicio percibida por los estudiantes universitarios, donde el mayor porcentaje se encuentra en el nivel alto con un $71,13 \%$.

\section{Gráfico 3}

\section{Histograma de la dimensión Fiabilidad}

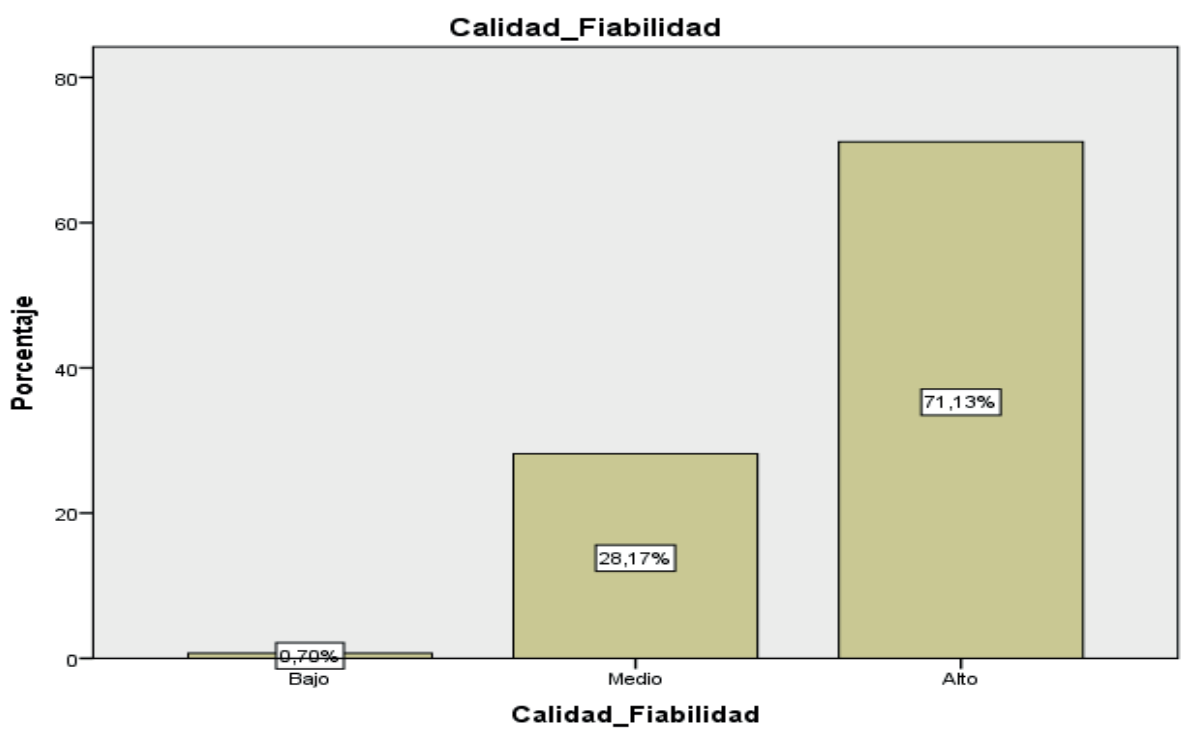

Fuente: Elaboración propia (2021) 
Neyra-Huamani, Lidia; Flores-Morales, Jorge Alberto; Pacheco-Pomarino, Mariluz Calidad de servicio en tiempos de Covid-19 en estudiantes universitarios

En la tabla 4 la dimensión Capacidad de respuesta de la variable calidad de servicio, de 142 estudiantes que representan el $100 \%$ se evidencia 88 estudiantes que representa el 62
$\%$ alcanzó el nivel alto. Además 54 estudiantes se encuentran en el nivel medio con un $38 \%$ de calidad de respuesta. En esta dimensión, ningún estudiante se encuentra en el nivel bajo.

Tabla 4

Dimensión 3: Capacidad de respuesta

\begin{tabular}{|c|c|c|c|c|c|}
\hline & & Frecuencia & Porcentaje & Porcentaje válido & $\begin{array}{l}\text { Porcentaje } \\
\text { acumulado }\end{array}$ \\
\hline \multirow[t]{3}{*}{ Válido } & Medio & 54 & 38,0 & 38,0 & 38,0 \\
\hline & Alto & 88 & 62,0 & 62,0 & 100,0 \\
\hline & Total & 142 & 100,0 & 100,0 & \\
\hline
\end{tabular}

Fuente: Elaboración propia (2021)

El gráfico 4 representa los datos de la dimensión Capacidad de respuesta en la Calidad de servicio percibida por los estudiantes universitarios, donde el mayor porcentaje se encuentra en el nivel alto con un $62 \%$.

\section{Gráfico 4 \\ Histograma de la dimensión Capacidad de respuesta}

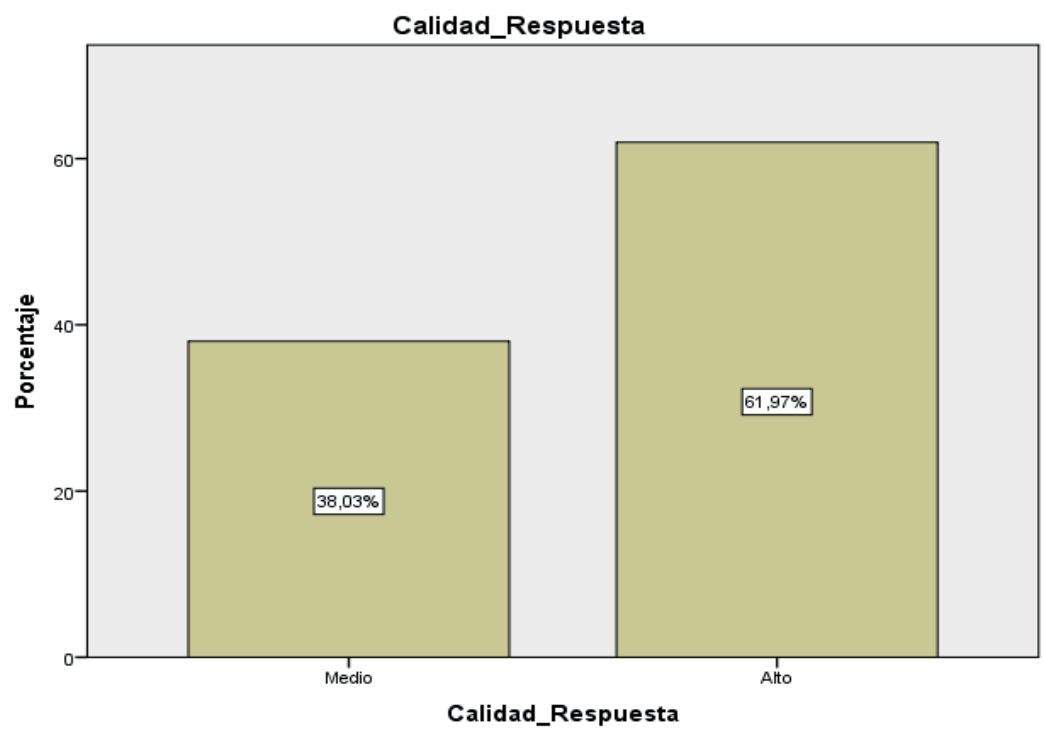

Fuente: Elaboración propia (2021) 
En la tabla 5 la dimensión Seguridad de los 142 estudiantes que representan el $100 \%$ se evidencia 86 estudiantes consideran que en la dimensión Seguridad de la Calidad de servicio se encuentra en un nivel alto representado un 60,56 \% Además 54 estudiantes se encuentran en el nivel promedio con un $38,03 \%$ y 2 estudiantes lo perciben en un nivel bajo con un 1,41 $\%$ en la dimensión Seguridad.

Tabla 5

Dimensión 4: Seguridad

\begin{tabular}{|c|c|c|c|c|c|}
\hline & & Frecuencia & Porcentaje & Porcentaje válido & $\begin{array}{l}\text { Porcentaje } \\
\text { acumulado }\end{array}$ \\
\hline \multirow[t]{4}{*}{ Válido } & Bajo & 2 & 1,4 & 1,4 & 1,4 \\
\hline & Medio & 54 & 38,0 & 38,0 & 39,4 \\
\hline & Alto & 86 & 60,6 & 60,6 & 100,0 \\
\hline & Total & 142 & 100,0 & 100,0 & \\
\hline
\end{tabular}

Fuente: Elaboración propia (2021)

El gráfico 5 representa los datos de la dimensión Seguridad en la Calidad de servicio percibida por los estudiantes universitarios, donde el mayor porcentaje se encuentra en el nivel alto con un $60,56 \%$.

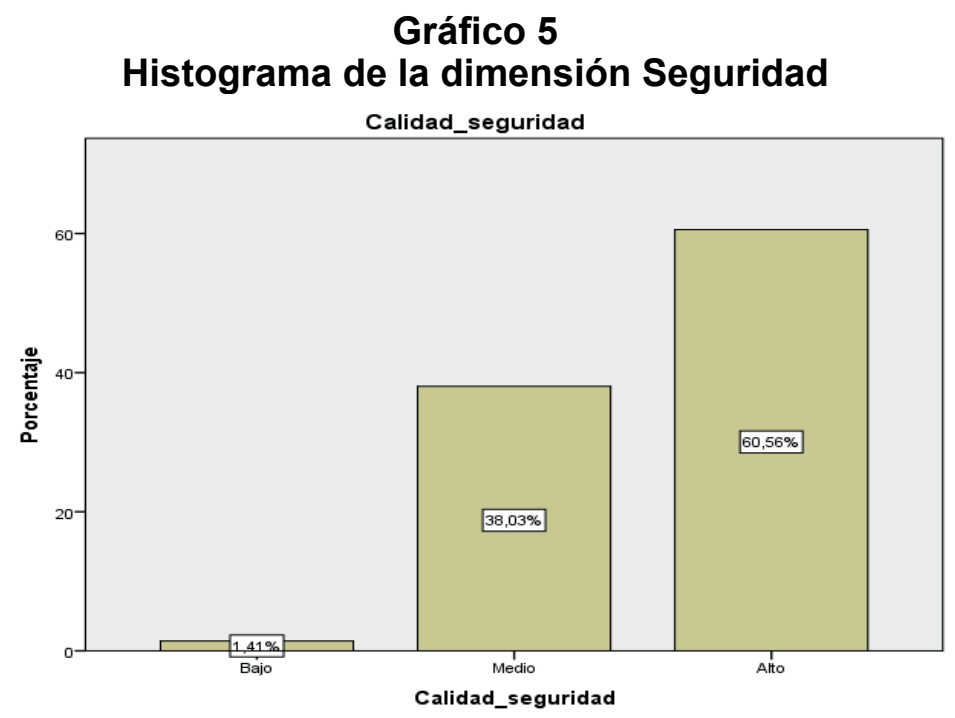

Fuente: Elaboración propia (2021) 
Neyra-Huamani, Lidia; Flores-Morales, Jorge Alberto; Pacheco-Pomarino, Mariluz Calidad de servicio en tiempos de Covid-19 en estudiantes universitarios

En la tabla 6 la dimensión empatía de la variable calidad de servicio, de 142 estudiantes que representan el $100 \%$ se evidencia 84 estudiantes que representa el 59,2 \% alcanzó el nivel alto. Asimismo,
54 estudiantes se encuentran en el nivel medio con un $38 \%$ y 4 estudiantes se encuentran en un nivel bajo con un 2,8 $\%$ en la dimensión empatía.

\section{Tabla 6}

\section{Dimensión 5: Empatía}

\begin{tabular}{rlrrrrr}
\hline & & \multicolumn{2}{c}{ Frecuencia } & Porcentaje & Porcentaje válido & \multicolumn{2}{c}{$\begin{array}{r}\text { Porcentaje } \\
\text { acumulado }\end{array}$} \\
\hline \multirow{3}{*}{ Válido } & \multicolumn{1}{c}{ Bajo } & 4 & 2,8 & 2,8 & 2,8 \\
\cline { 2 - 6 } & Medio & 54 & 38,0 & 38,0 & 40,8 \\
\cline { 2 - 6 } & Alto & 84 & 59,2 & 59,2 & 100,0 \\
\cline { 2 - 6 } & Total & 142 & 100,0 & 100,0 & \\
\hline
\end{tabular}

Fuente: Elaboración propia (2021)

El gráfico 6 representa los datos de la dimensión Empatía en la Calidad de servicio percibida por los estudiantes universitarios, donde el mayor porcentaje se encuentra en el nivel alto con un $59,15 \%$.

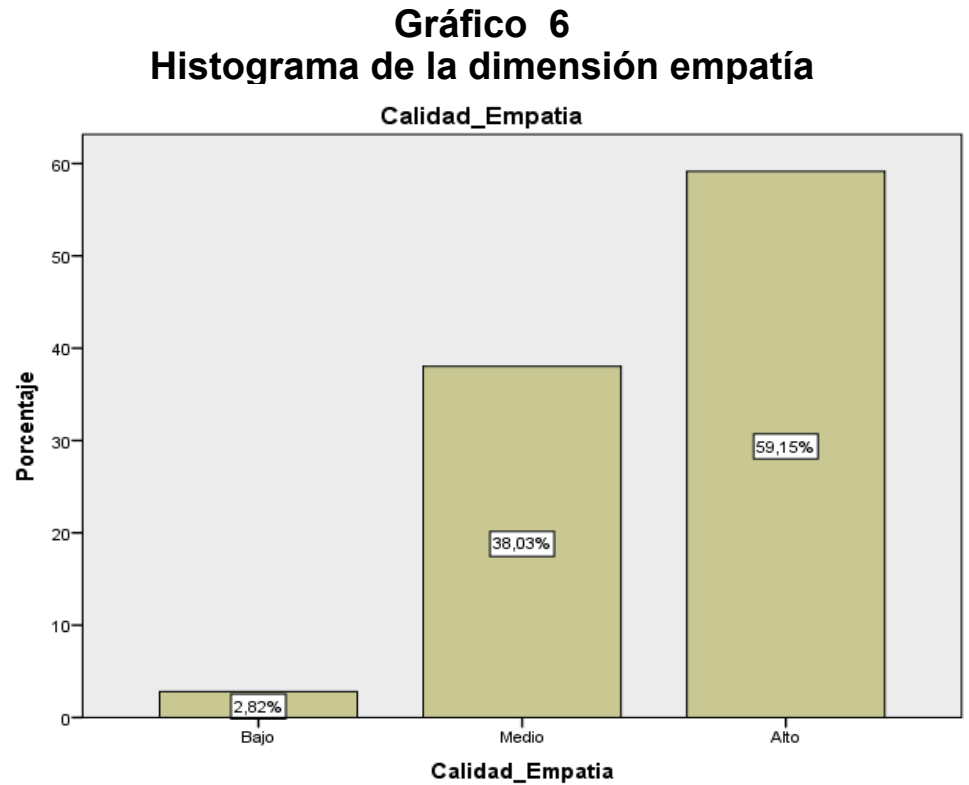

Fuente: Elaboración propia (2021) 
Los resultados obtenidos en el presente estudio proporcionan información sobre la percepción de calidad de servicio en tiempos de pandemia en los estudiantes de una universidad nacional de Lima. Estos resultados muestran que la mayoría de los estudiantes $66,9 \%$ tienen una alta percepción de calidad de servicio. Asimismo, un 33,1 \% tienen una percepción de nivel medio de calidad de servicio y ninguna en el nivel bajo. Similar resultado se demostró en la variable calidad de servicio. En el cual, el $80 \%$ de los estudiantes perciben como Bueno y Muy Bueno, el nivel de la calidad de los servicios prestados por dichos procesos (Castañeda, Benítez, \& Parra, 2019).

Los resultados obtenidos por Cervantes, et al, sobre la calidad de los servicios en una institución educativa a nivel superior son buenas. En este caso los autores señalan que los servicios vayan acompañados de un procedimiento de calidad que permita una atención respetuosa, ágil y oportuna y a la vez llene las expectativas de los clientes a través de un servicio o producto con valor agregado y diferenciador (Cervantes, et al, 2018).

En cambio, en una institución de salud tuvieron como resultado sobre la percepción de la calidad del servicio por parte de los médicos un nivel bajo en un $7,3 \%$, nivel medio un $54 \%$ y nivel alto un 38,7\% (Podestá \& Maceda, 2018). En el cual, hay un nivel medio en la percepción de la calidad de servicio.

En el presente estudio en la dimensión Tangibilidad de la variable calidad de servicio, se evidencia el predominio de un nivel medio con $52,1 \%$ de percepción de tangibilidad. Seguido del nivel alto de percepción con un
$45,1 \%$ y un nivel bajo con un $2,8 \%$ en la dimensión tangibilidad. Estos resultados difieren de otra institución educativa. En el cual, obtuvieron en la evaluación del estado de la calidad de las instalaciones de los servicios del campus un bajo nivel de satisfacción de los estudiantes, lo que se desprende de la brecha existente entre las percepciones y las expectativas de los usuarios, donde para cada ítem hay un resultado negativo. Es decir, la calidad de las instalaciones de servicios del campus sigue estando muy por debajo de las expectativas de los usuarios (Napitupulu et al, 2018).

De igual forma, la dimensión Tangibilidad de la variable calidad de servicio, se evidencia el predominio de un nivel medio con 52,1\%. Además, la dimensión seguridad el 60,6 \% alcanzó el nivel alto. Y la dimensión empatía alcanzó un nivel alto con 59,2 \%. Estos resultados difieren del siguiente estudio. En el cual, las dimensiones evaluadas para la calidad de servicio como la tangibilidad, seguridad, responsabilidad y empatía evidenciaron niveles bajos en un $47,1 \%, 50 \%, 41,4 \%$ y $51.4 \%$ consecutivamente (Chuquicusma et al, 2020)

\section{Conclusiones}

La pandemia ha traído nuevos retos al proceso de enseñanza aprendizaje por el cual las universidades han tenido que diversificar su atención de una modalidad presencial a otra virtual manteniendo igual o mejor la calidad de servicios en sus usuarios que son principalmente los estudiantes.

- La calidad de servicio en su dimensión elementos tangibles se expresan a través de los Campus o plataformas virtuales con lo que 
Neyra-Huamani, Lidia; Flores-Morales, Jorge Alberto; Pacheco-Pomarino, Mariluz

Calidad de servicio en tiempos de Covid-19 en estudiantes universitarios

cuenta la universidad, en la cual los alumnos por diferentes medios sean Tables, celular, laptop, etc. logran accesibilidad a los servicios estudiantiles como la biblioteca virtual, consejería académica y a sus clases.

- La calidad de servicio en su dimensión fiabilidad se expresa en la capacidad de respuesta que tanto los docentes como el personal que atiende los requerimientos del estudiante respondan prontamente y perciba el interés genuino de solucionar el impase presentado.

- La calidad de servicio en su dimensión capacidad de respuesta, señala que los estudiantes perciben que hay voluntad tanto de sus maestros como del personal administrativo de ofrecer un servicio con prontitud y con buena disposición de ánimo.

- Con respecto, a la calidad de servicio en su dimensión seguridad expresa que la percepción por parte de los estudiantes resulta positiva en cuanto que se siente seguros que su universidad está preocupada por su enseñanza en cuanto se implementa los soportes y ayuda para la ejecución de una enseñanza remota y hay respuestas honestas ante dudas que se les presentan en el desarrollo de su aprendizaje.

- La calidad de servicio en su dimensión empatía implica que el estudiante perciba la preocupación de la casa de estudio superior en capacitarle en el uso de las plataformas educativas (Microsoff Team, Zoom, Canva, etc) y el trato amable de los miembros de la comunidad universitaria la manera de como conectarse con sus docentes en los diferentes cursos que lleva. Por ello es importante las netiquetas, es decir las normas de comportamiento entre los usuarios de las redes que han de estar siempre presentes generando el espacio de una retroalimentación positiva e identificación.

Finalmente, es importante medir siempre la calidad de servicio educativo y más una en estos tiempos de pandemia, para poder hacer las modificaciones debidas o a tiempo y mantener el buen ambiente de la comunidad universitaria expresada en la virtualidad.

\section{Referencias bibliográficas}

Castañeda, E. D. C., Benítez, A. M. F., \& Parra, A. B. S. (2019). Analysis of the quality of academic-administrative services in a Colombian university. Revista Lasallista de Investigacion, 16(1), 47-60. https://doi. org/10.22507/rli.v16n1a9

CEPAL-UNESCO. (2020). La educación en tiempos de la pandemia de COVID-19. Geopolitica(S), 11, $1 . \quad$ https://www.cepal.org/es/ publicaciones/45904-la-educaciontiempos-la-pandemia-covid-19

Cervantes, V., Stefanell, Í., Peralta, P., \& Salgado, R. (2018). Calidad de Servicio en una institución de educación superior en la ciudad de Barranquilla. Ciencias Administrativas, (11), 017. https://doi. org/10.24215/23143738e017

Chuquicusma, T., Luján, P., SánchezChero, M., Montalban, Y., Silva, R., \& Rosas-Prado, C. (2020). Calidad de servicio y nivel de satisfacción en el hospital José Cayetano Heredia, Perú. Revista de La Universidad Del Zulia, 11(31), 39-51. https://doi. org/10.46925//rdluz.31.04

Datta, K. S., \& Vardhan, J. (2017). A 
SERVQUAL-based framework for assessing quality of international branch campuses in UAE: A management students' perspective. SAGE Open, 7(1). https://doi. org/10.1177/2158244016676294

Dwidienawati, D., Abdinagoro, S. B., Tjahjana, D., \& Gandasari, D. (2020). Volume 9 International Journal of Advanced Trends in Computer Science and Engineering Available Online at http://www. warse.org/IJATCSE/static/pdf/file/ ijatcse93922020.pdf

Hernández, R., \& Mendoza, C. (2018). Las rutas Cuantitativa Cualitativa y Mixta. In Metodología de la investigación. Las rutas cuantitativa, cualitativa y mixta.

Kinker, P., Swarnakar, V., Jain, R., y Singh, A. (2021). A QFD-TISM approach for service quality improvement in polytechnic education institutes: a case study. International Journal of Applied Systemic Studies, 9(2), 85-113. https://doi.org/10.1504/ $\underline{\text { IJASS.2020.10034539 }}$

Lewis, R. ., \& Mitchell, V. (1990). Defining and Measuring the Quality of Customer Service. Marketing Intelligence \& Planning, 8(6), 11-17. https://doi.org/10.1108/ EUM0000000001086

Mejías, A., Valle, M., \& Vega, A. (2016). La calidad de los servicios universitarios: reflexiones a partir del estudio de casos en el contexto latinoamericano. Industrial Data, 16(2), 13. https://doi.org/10.15381/ idata.v16i2.11869

Moser, K. M., Wei, T., \& Brenner, D. (2021). Remote teaching during COVID-19: Implications from a national survey of language educators. System, 97, 102431. https://doi.org/10.1016/i. system. 2020.102431
Napitupulu, D. ., Rahim, R. ., Abdullah, D. ., Setiawan, M. ., Abdillah, L. ., Ahmar, A. ., ... Pranolo, A. . (2018). Analysis of Student Satisfaction Toward Quality of Service Facility Analysis of Student Satisfaction Toward Quality of Service Facility.

Parasuraman, A., Zeithaml, A. ., \& Berry, L. (1988). SERVQUAL: A multipleitem scale for measuring consumer perceptions of service quality. Journal of Retailing, 64(1), 12-40.

Parasuraman, A., Zeithaml, V. A., \& Berry, L. L. (1985). A Conceptual Model of Service Quality and Its Implications for Future Research. Journal of Marketing, 49(4), 41. https://doi.org/10.2307/1251430

Podestá, L., \& Maceda, M. (2018). Calidad percibida del servicio y cultura de seguridad en salud en el personal médico del Hospital II EsSalud Vitarte. Lima, 2017. Horizonte Médico (Lima), 18(3), 48-56. http://dx.doi.org/10.24265/ horizmed.2018.v18n3.08

Rojas-Martínez, C., Niebles-Nuñez, W., Pacheco-Ruíz, C., \& HernándezPalma, H. G. (2020). Calidad de servicio como elemento clave de la responsabilidad social en pequeñas y medianas empresas. Información Tecnológica, 31(4), 221-232. https://doi.org/10.4067/s0718$\underline{07642020000400221}$

Tan, C. M., \& Goh, T. N. (2017). Theory and practice of quality and reliability engineering in Asia industry. Theory and Practice of Quality and Reliability Engineering in Asia Industry, 1-300. https://doi.org/10.1007/978-981-103290-5

Tejedor, S., Cervi, L., Pérez-Escoda, A., Tusa, F., \& Parola, A. (2021). Higher education response in the time of coronavirus: Perceptions of teachers and students, and open innovation. 
Neyra-Huamani, Lidia; Flores-Morales, Jorge Alberto; Pacheco-Pomarino, Mariluz Calidad de servicio en tiempos de Covid-19 en estudiantes universitarios

Journal of Open Innovation: Technology, Market, and Complexity, 7(1), 1-15. https://doi.org/10.3390/ joitmc7010043

Wisniewski, M. (2001). Using Serqual to assess customer satisfaction with public sector services. Managing Service Quality: An International Journal, 11(6), 380-388.
Yarimoglu, E. K. (2014). A Review on Dimensions of Service Quality Models. Journal of Marketing Management, 2(2), 79-93. 